\title{
Alternativen zu Mini- und Midijobs? Die Beispiele Frankreich und Vereinigtes Königreich
}

Mini- und Midijobs haben einen steuer- und sozialversicherungsrechtlichen Sonderstatus mit dem Ziel, Beschäftigung durch monetäre Anreize für Beschäftigte zu erhöhen. Damit sollen sie eine Brückenfunktion in reguläre Beschäftigung haben, was sich in der Realität allerdings nicht zeigt. Deshalb wird hier der Frage nachgegangen, welche Regelungen für Beschäftigungsverhältnisse im unteren Einkommensbereich in anderen Ländern existieren, wie erfolgreich diese sind und welche Lehren daraus für Deutschland gezogen werden können. ${ }^{\circ}$

ALEXANDER HERZOG-STEIN, WERNER SESSELMEIER

\section{Einleitung}

Mit der Neuregelung der geringfügigen Beschäftigung durch die Einführung der Mini- und Midijobs im Jahr 2003 verband die Politik drei Ziele: Erstens, sollten Beschäftigungsmöglichkeiten im Niedriglohnsektor geschaffen werden (Bundesregierung 2003). Zweitens, sollten Minijobs für Arbeitslose eine Brücke in ein sozialversicherungspflichtiges Beschäftigungsverhältnis darstellen (BMWA 2003). Drittens, sollte die Schwarzarbeit vor allem in Privathaushalten eingedämmt werden (Bundesregierung 2003). ${ }^{\boldsymbol{Q}}$ Mit den Midijobs wurde zudem explizit auch abgabentechnisch ein fließender Übergang zwischen Minijobs und sozialversicherungspflichtiger Beschäftigung geschaffen. Durch die Abschaffung der vormals für die geringfügige Beschäftigung geltenden Arbeitszeitbegrenzung auf maximal 15 Wochenstunden und das Fehlen eines allgemeinen gesetzlichen Mindestlohns wurden die Mini- und Midijobregelungen die entscheidenden steuer- und abgabenrechtlichen Rahmenbedingungen für Beschäftigungsverhältnisse mit niedrigen Erwerbseinkommen, sofern kein Anspruch auf Unterstützung durch die Grundsicherung für Arbeitsuchende (Arbeitslosengeld II) besteht.

Aus ökonomischer Sicht handelt es sich sowohl bei den Mini- als auch den Midijobs um arbeitnehmerseitige Lohnzuschüsse, weil Arbeitseinkommen bis $400 €$ ganz und bis $800 €$ mit abnehmender Rate von der Zahlung von Steuern und Sozialabgaben befreit sind. Diese niedrigen Erwerbseinkommen können aufgrund niedriger Stundenlöhne oder eines geringen Stundenumfangs oder von beidem zustande kommen. In der Praxis haben sich die Mini- und Midijobs aber nicht bewährt. Sie haben sich nicht als zielführend erwiesen und stattdessen erhebliche Probleme und unerwünschte Effekte mit sich gebracht. Die Folge ist, dass in Deutschland Handlungsbedarf in Bezug auf das Steuer- und Abgabensystem im unteren Einkommensbereich besteht (vgl. Weinkopf 2011).

In diesem Beitrag wird der Frage nachgegangen, wie im Vereinigten Königreich und in Frankreich, zwei großen Volkswirtschaften in der Europäischen Union, die ebenfalls Lohnzuschüsse für niedrige Entgelte gewähren, das Steuerund Abgabensystem im unteren Einkommensbereich ausgestaltet ist. Auch wenn eine Übertragbarkeit länderspezifischer Systeme und Institutionen nur bedingt möglich ist, so sollen doch durch die Betrachtung dieser beiden Länder zumindest einige Hinweise auf mögliche Alternativen gewonnen werden. Da Lohnzuschussmodelle auf eine Beschäftigungserhöhung bzw. eine Einkommenserhöhung abzielen, liegt

\footnotetext{
(1) Die Autoren danken Christian Pirzer für seine Unterstützung.

(2) Für Details siehe u.a. Apel et al. (2006), S. 106. Das dritte Ziel lassen wir im Folgenden außer Acht.
} 
unser Betrachtungsschwerpunkt auf der Abgabenbelastung niedriger Einkommen und den damit verbundenen Anreizen zur Arbeitsaufnahme sowie der Einkommenssituation von Personen mit niedrigem Einkommen.

Hierzu werden zunächst die Mini- und Midijobregelungen im Kontext des deutschen Abgabensystems beschrieben und einige bestehende Probleme und Nebeneffekte herausgearbeitet (Abschnitt 2). Danach werden die existierenden Regelungen im Hinblick auf Beschäftigungsverhältnisse im unteren Einkommensbereich im Vereinigten Königreich (3) und in Frankreich (4) dargestellt. Abschnitt 5 skizziert die Beschäftigungs- und Einkommenseffekte dieser Regelungen. Abschließend werden einige Schlussfolgerungen für Deutschland gezogen (6). ${ }^{\circledR}$

\section{Steuer- und sozialversicherungs- rechtlicher Sonderstatus}

\subsection{Abgabenbelastung im unteren Einkommensbereich}

Arbeitnehmer und Arbeitnehmerinnen entrichten auf Einkommen aus einem Minijob - auch wenn der Minijob im Nebenerwerb zusätzlich zu einer sozialversicherungspflichtigen Hauptbeschäftigung erfolgt - keine arbeitnehmerseitigen Sozialversicherungsbeiträge und (in der Regel) auch keine Einkommensteuer. Arbeitgeber zahlen dagegen neben dem Direktlohn gegenwärtig Pauschalabgaben in Höhe von 30,74\% des Verdienstes an die Minijob-Zentrale. Damit betragen die Arbeitskosten bei einem Minijob in Höhe von $400 €$ für den Arbeitgeber 520,96 €. Bei Minijobs in Privathaushalten sind die Abgaben geringer. Hier zahlen Arbeitgeber nur 14,34\% des Verdienstes an die Minijob-Zentrale, sodass die gesamten Arbeitskosten bei wiederum $400 €$ Lohnhöhe insgesamt 457,36€ betragen.

Im Anschluss an die Minijobs gibt es einen Entgeltkorridor von $400 €$ bis $800 €$, die sogenannten Midijobs, in dem der Arbeitnehmerbeitrag zu den Sozialversicherungen ermäßigt ist. Der Beitragssatz startet bei $11 \%$ und erreicht $21 \%$ bei $800 €$, wobei er nicht linear ansteigt (SVR 2006, Ziffer 89, S. 71). Für Arbeitgeber entfallen dagegen mit dem Übergang vom Mini- in den Midijobbereich die Pauschalabgaben; hier sind stattdessen volle Arbeitgeberbeitragssätze zu den Sozialversicherungen in Höhe von knapp 20 \% zu entrichten. Wie von der Politik angestrebt, wird mit dieser Gleitzone die Grenzabgabenbelastung für die Erwerbstätigen beim Übergang zur sozialversicherungspflichtigen Beschäftigung im Vergleich zu früher gemildert.

Die 400-€-Grenze der Minijobs ist kein Freibetrag, sondern eine Freigrenze. Somit wird ein Beschäftigungsverhältnis beim Übergang vom Mini- in den Midijobbereich nicht nur (reduziert) sozialversicherungs-, sondern voll steuerpflichtig. Je nach Haushaltskonstellation wirkt sich diese Regelung unterschiedlich aus: Bei einem Einperso- nenhaushalt mit einem Steuergrundfreibetrag von $8.004 €$ im Jahr 2011 und einem Eingangssteuersatz von $14 \%$ spielt das im Normalfall keine Rolle. Bei Zweitverdienern, die steuerlich gemeinsam mit ihren Ehepartnern veranlagt werden, sieht die Situation jedoch anders aus: Der Minijob ist in der Regel weiterhin steuer- und abgabenfrei. Oberhalb dieser Freigrenze von $400 €$ bezieht sich die Steuerlast von Zweitverdienern aber auf den gesamten Verdienst ab dem ersten Euro, sofern das gemeinsame Haushaltseinkommen über dem Grundfreibetrag für Paare (16.008 €) liegt. Fällig wird dabei zudem der in der Regel höhere Durchschnittssteuersatz des Ehepaares und nicht der Eingangssteuersatz. Die Folge ist ein sprunghafter Anstieg der Steuerbelastung beim Übergang vom steuer- und beitragsfreien Mini- zum Midijob.

\subsection{Effekte der Abgabenregelungen}

In der Realität sind verschiedene Probleme zwischen den politisch formulierten Zielen und den eingetretenen Folgen auszumachen.

(1) Es gibt deutliche Anzeichen dafür, dass die Abgabenzahllast der Arbeitgeber für Minijobs in Höhe von gut 30 \% nicht der tatsächlichen Traglast, d.h. der effektiven Belastung durch Steuern oder Abgaben, entspricht. Entscheidend aus ökonomischer Sicht ist aber die Traglast. Vor dem Hintergrund des Zusammenhangs, dass die Traglast der Pauschalabgaben umso mehr bei den Beschäftigten liegt, je inelastischer das Arbeitsangebot in Relation zur Arbeitsnachfrage im unteren Entgeltbereich ist (siehe beispielsweise Nicholson 1998, S. $444 \mathrm{ff}$.), lässt die starke Zunahme der Minijobs und der auffällig hohe Niedriglohnanteil unter den Minijobberinnen und Minijobbern (Voss/Weinkopf in diesem Heft; Wingerter 2009) darauf schließen, dass es den Arbeitgebern in erheblichem Umfang gelingt, die Pauschalabgaben im Minijobbereich auf die dort Beschäftigten überzuwälzen und die Arbeitskosten entsprechend zu senken. Damit tragen die Arbeitnehmer die Last der Pauschalabgabe der Arbeitgeber.

(2) Die Ausgestaltung der Mini- und Midijobregelungen setzt Anreize, dass insbesondere Zweitverdiener in der sogenannten „Geringfügigkeitsfalle“ verbleiben. Für sie ist eine Ausweitung der Arbeitszeit oder eine besser entlohnte höherwertige Tätigkeit jenseits des Minijobs aufgrund des sprunghaften Anstiegs der Steuer- und Beitragsbelastung beim Übergang vom steuer- und beitragsfreien Mini- zum Midijob wenig attraktiv (Eichhorst/Thode 2010, S. 36f.; Bäcker/Neuffer in diesem Heft). Durch die „Geringfügigkeitsfalle“ ist eine Brückenfunktion der Minijobs in Normalarbeitsverhältnisse zweifelhaft.

(3) Die nachfolgenden Ausführungen konzentrieren sich auf Haushalte ohne Kinder. Aus Gründen der Vergleichbarkeit werden die Steuer- und Abgabenregelungen 2008 benutzt. 
(3) Die Minijobregelung fördert eine Ausweitung des Arbeitsangebots von bereits sozialversicherungspflichtig beschäftigten Personen, da zusätzliche bezahlte Arbeitsstunden im Rahmen eines Minijobs im Nebenerwerb steuer- und abgabenfrei sind. Dies führt zu potenziellen Konkurrenzsituationen um Arbeitsplätze mit gering qualifizierten oder anderweitig benachteiligten Arbeitsuchenden und reduziert möglicherweise die Arbeitsmarktchancen dieser Problemgruppen.

(4) Empfänger von Arbeitslosengeld (ALG) II (wie auch von ALG I) profitieren nur im Rahmen der Hinzuverdienstgrenzen von einem Mini- oder Midijob. Die nicht vorhandenen oder reduzierten Arbeitnehmerbeiträge zu den Sozialversicherungen bei den Mini- bzw. Midijobs haben keine finanziellen Auswirkungen für die ALG-II-Empfänger. Denn Veränderungen in der Abgabenbelastung auf das Bruttoeinkommen haben korrespondierende Änderungen des bezahlten Arbeitslosengeld II zur Folge, sodass höhere (niedrigere) Abgaben auf das Bruttoeinkommen zu höheren (niedrigeren) Transferleistungen führen (siehe SVR 2006, Ziffer 78, S. 66). Im Hinblick auf die Abgabenbelastung sind deshalb für Empfänger von ALG II die Transferentzugsraten in der Grundsicherung für Arbeitsuchende entscheidend. Da der Schwerpunkt des Beitrags die Rolle der Mini- und Midijobs im Rahmen des deutschen Steuer- und Abgabensystems ist, wird nachfolgend das Arbeitslosengeld II nicht weiter berücksichtigt, obwohl es sich aufgrund der Hinzuverdienstmöglichkeiten im Rahmen der Grundsicherung für Arbeitsuchende ebenfalls um einen Kombilohn handelt (vgl. Dingeldey et al. in diesem Heft).

All diese Folgen und Probleme der aktuellen Mini- und Midijobregelungen deuten darauf hin, dass in Deutschland Handlungsbedarf in Bezug auf das Steuer- und Abgabensystem im unteren Einkommensbereich besteht. Als möglicher Referenzpunkt werden deshalb im Folgenden die Regelungen, die im Vereinigten Königreich und in Frankreich im unteren Einkommensbereich existieren, dargestellt.

\section{Vereinigtes Königreich: Steuer- und Transfersystem im unteren Einkom- mensbereich}

Das Vereinigte Königreich, ein liberaler Wohlfahrtsstaat, hat ob seines Umgangs mit Erwerbseinkommen im unteren Einkommensbereich unter dem Slogan „Make Work Pay“ in den vergangenen Jahren erhebliche Aufmerksamkeit in Kontinentaleuropa erhalten (Bargain/Orsini 2006). Im Unterschied zu Deutschland und Frankreich ist das Steuerund Abgabensystem im Vereinigten Königreich durch individuelle Veranlagung, einen nur zweistufigen Steuertarif und niedrige Sozialversicherungsbeiträge gekennzeichnet. Im unteren Einkommensbereich liegt der Schwerpunkt auf arbeitnehmerseitigen Lohnzuschüssen in Form von Steuergutschriften (tax credits), ${ }^{\circledR}$ um niedrige Erwerbseinkommen finanziell aufzubessern, wobei Beschäftigungsverhältnisse explizit erst ab einem gewissen Stundenvolumen gefördert werden. Zudem gilt seit 1999 wieder ein allgemeiner gesetzlicher Mindestlohn; Ende 2008 betrug er 5,73£ $(7,20 €)$ pro Stunde. ${ }^{\ominus}$

Nach der Regierungsübernahme durch die Labour Party 1997 wurde arbeitnehmerseitigen Lohnzuschüssen in Form von Steuergutschriften eine bedeutsame Rolle in New Labours „Welfare to Work Strategy“ zugedacht (Strickland 1998), und so wurde ab 1999 das britische System der Steuergutschriften in mehreren Schritten verändert und ausgebaut. 2003 wurde die Steuergutschrift für Erwerbstätige, der Working Tax Credit (WTC), und damit das bis heute im Vereinigten Königreich existierende System an Steuergutschriften eingeführt (vgl. Kaltenborn/Knerr 2005). Der WTC ist eine Steuergutschrift, die Haushalte mit mindestens einem Erwerbstätigen und einem niedrigen Erwerbseinkommen in Form einer reduzierten Steuerschuld oder durch einen Transfer unterstützt und die explizit darauf abzielt, Personen für ihre gering entlohnte Erwerbstätigkeit finanziell zu belohnen. Im Unterschied zu früher haben auch kinderlose Haushalte generell Anspruch auf den WTC. Die tatsächliche Anspruchsberechtigung und die Höhe der Leistungen sind abhängig vom Umfang der gewöhnlich geleisteten wöchentlichen Arbeitsstunden, der Bedürftigkeit des Haushalts gemessen anhand der Höhe des Haushaltseinkommens und der Existenz von Kindern im Haushalt. Wie in Frankreich (vgl. Abschnitt 4) gilt im Vereinigten Königreich eine Mindestförderschwelle für den arbeitnehmerseitigen Lohnzuschuss: Anspruchsberechtigt sind Haushalte mit Kindern, wenn die Antragsteller mindestens 16 Jahre alt sind und einer Erwerbstätigkeit im Umfang von mindestens 16 Wochenstunden nachgehen, oder kinderlose Haushalte, wenn Antragsteller älter als 25 sind und die Erwerbstätigkeit üblicherweise ein Volumen von mehr als 30 Wochenstunden aufweist. Im Steuerjahr 2008/09 setzte sich der WTC aus einem Grundbetrag in Höhe von jährlich $1.800 £(2.261 €)^{\ominus}$ und einem Alleinerziehenden- oder

\footnotetext{
(4) Eine Steuergutschrift ist nicht zu verwechseln mit Steuerfreibeträgen, die die Höhe des zu versteuernden Einkommens reduzieren. Bei einer Steuergutschrift wird die Steuerschuld um die entsprechende Höhe der Gutschrift reduziert. Übersteigt die Höhe der Steuergutschrift die Steuerschuld, so wird die Differenz wie bei einer negativen Einkommensteuer in Form eines Transfers ausbezahlt.

(5) Aktuell liegt der Mindestlohn bei 6,08 $£$ (= Britisches Pfund, GBP), was 7,64 € entspricht.

(6 Die Umrechnung in Euro erfolgt mittels des von Eurostat veröffentlichten jahresdurchschnittlichen Wechselkurses 2008: Ein Euro entsprach GBP 0,79628. Die Beträge werden jeweils auf ganze Euro gerundet, soweit im Text nicht explizit auch Cent angegeben werden.
} 
ABB.

\section{Steuergutschrift für Erwerbstätige im Vereinigten Königreich (Working Tax Credit) 2008* \\ Angaben in Euro $\quad$ Einzelperson Einverdienerhaushalt}

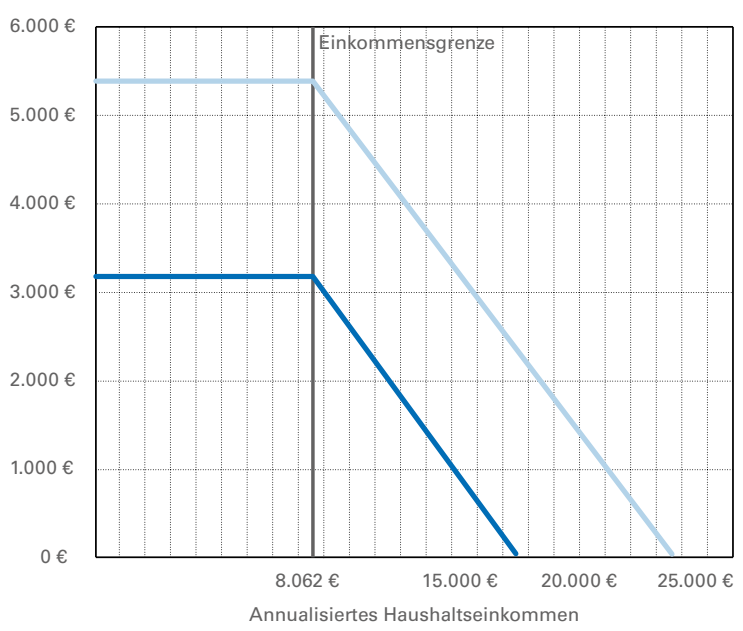

* berücksichtigt sind Grundbetrag, 30-Stunden-Element und Partnerelement; $1 €=£ 0,79628$.

Quelle: Darstellung der Autoren

Partnerelement in Höhe von $1.770 £(2.223 €)$ zusammen. $^{\circ}$ Beträgt die gewöhnlich geleistete Zahl der Arbeitsstunden des Haushalts mehr als 30 Stunden - wobei zumindest ein erwachsenes Haushaltsmitglied mindestens einer abhängigen Beschäftigung im Umfang von 16 Wochenstunden nachgehen muss -, dann erhöht sich die maximal mögliche Steuergutschrift um jährlich $735 £(923 €){ }^{8}$ Insgesamt kann somit bei einem erwerbstätigen Zweipersonenhaushalt die Steuergutschrift $4.305 £(5.407 €)$ im Jahr erreichen. Hat der Haushalt aufgrund seines niedrigen Erwerbseinkommens keine Steuern zu entrichten, so erhält er diese Summe als Transfer ausbezahlt. Ab einem jährlichen Haushaltseinkommen über der Einkommensgrenze von $6.420 £(8.062 €)$ wird die Steuergutschrift mit einer Transferentzugsrate von $39 \%$ reduziert (Abbildung 1).

Nach Schätzungen der britischen Finanzbehörde beliefen sich die Ausgaben für den WTC auf rund 7,5 Mrd. $£$ (9,4 Mrd. €) im Steuerjahr 2008/09 (HM Revenue and Customs 2009, Tabelle 3.1).

Der WTC kann nicht losgelöst vom übrigen Steuerund Transfersystem des Vereinigten Königreichs betrachtet werden. So sind für die Grenzsteuerbelastung neben der Transferentzugsrate auch die Höhe des Grenzsteuersatzes und der Sozialversicherungsbeiträge von Relevanz. Bedeutsam ist ebenfalls, dass im Vereinigten Königreich im Unterschied zu Deutschland und Frankreich das Steuersystem im Prinzip voll individualisiert ist (Dingeldey 2002, S. 155). Von diesem Grundsatz wird jedoch teilweise abgewichen, da für Steuergutschriften wie dem WTC das Haushaltseinkommen die Bemessungsgrundlage bildet (Kaltenborn/Knerr 2005, Tabellen 56 und 61). Der persönliche Steuerfreibetrag lag im Steuerjahr 2008/09 bei $6.035 £$ (7.579 €). Für Einkommen oberhalb des Grundfreibetrages gilt ein einheitlicher Grenzsteuersatz in Höhe von $20 \%$. Dieser Grundsteuersatz wird auf zu versteuerndes Einkommen bis $34.800 £(43.703 €)$ angewendet. Oberhalb dieser Einkommensgrenze gilt der höhere Einkommensteuersatz von $40 \%$.

Bei den Sozialversicherungsbeiträgen - es handelt sich hierbei nur um Beiträge zur Renten- und Arbeitslosenversicherung, da das Gesundheitswesen im Vereinigten Königreich steuerfinanziert ist - ist zwischen Arbeitnehmerund Arbeitgeberbeiträgen zu unterscheiden. Die Arbeitnehmerbeiträge betragen $11 \%$ des wöchentlichen Einkommens zwischen $105 £$ ( $5.475 £$ oder $6.876 € \mathrm{im} \mathrm{Jahr}$ ) und $770 £$ (40.150 oder $50.422 € \mathrm{im} \mathrm{Jahr)} \mathrm{plus} 1 \%$ des Einkommens über $770 £$. Die Arbeitgeberbeiträge betragen 12,8 \% für wöchentliche Einkommen über $105 \mathrm{£}$. Für wöchentliche Einkommen bis $105 £$ fallen keine Sozialversicherungsbeiträge an. Im Vereinigten Königreich ist also wie in Deutschland Beschäftigung mit einem „geringfügigen“ Einkommen sozialversicherungsfrei. Im Unterschied zu Deutschland werden Arbeitgeber und Arbeitnehmer durch die Freibeträge in den Sozialversicherungen nominal entlastet. Die Einkommensgrenze von umgerechnet $132 €$ pro Woche liegt über der deutschen Minijobschwelle von rund $92 €$ pro Woche. Gleichzeitig besteht aber für solche Beschäftigungsverhältnisse aufgrund von Mindestlohn und Mindestförderschwelle kein Anspruch auf den WTC. Somit gehen von dem Freibetrag in den Sozialversicherungen und der Mindestförderschwelle widersprüchliche Anreize aus.

Zusammenfassend ist der relativ großzügige Lohnzuschuss in Form von Steuergutschriften zur direkten finanziellen Unterstützung niedriger Erwerbseinkommen und zur Erhöhung der Arbeitsanreize auffällig. Lohnzuschüsse erhalten nur Beschäftigte oberhalb einer Mindestförderschwelle von 16 bzw. 30 Wochenarbeitsstunden. Nicht zu vernachlässigen ist dabei die flankierende Wirkung des allgemeinen gesetzlichen Mindestlohns, der eine Lohnuntergrenze definiert und verhindert, dass durch den umfassenden Lohnzuschuss das Lohngefüge nach unten ins Rutschen kommt.

$(7$ Zum Vergleich: Das Arbeitseinkommen des durchschnittlichen Arbeitnehmers lag nach Berechnungen der OECD im Jahr 2008 bei $33.473 £(42.037 €)$.

(8 Darüber hinaus gibt es weitere zusätzliche Beiträge im Rahmen des WTC für bestimmte Umstände oder Personengruppen. Diese Extrabeträge werden aber hier nicht weiter berücksichtigt. 


\section{Frankreich: Steuer- und Transfersys- tem im unteren Einkommensbereich}

Frankreich ist neben Deutschland der zweite große Vertreter der konservativen Wohlfahrtsstaaten. Im Hinblick auf das Steuer- und Transfersystem im unteren Einkommensbereich zeichnet sich das französische System durch eine Doppelstrategie aus: Einerseits setzt es auf eine arbeitgeberseitige Subventionierung der Sozialversicherungsbeiträge und andererseits auf einen arbeitnehmerseitigen Lohnzuschuss. Zudem gilt in Frankreich, wie im Vereinigten Königreich, ein allgemeiner gesetzlicher Mindestlohn, der salaire minimum interprofessionnel de croissance (SMIC). Der SMIC wird jährlich erhöht und betrug 2008 8,71 € pro Stunde. ${ }^{\ominus}$

Ähnlich wie in Deutschland sind Sozialversicherungsbeiträge von Arbeitnehmern und Arbeitgebern tragende Säulen der Finanzierung des französischen Wohlfahrtsstaates. Folglich sind die Arbeitskosten in Frankreich und Deutschland gleichermaßen durch hohe Lohnnebenkosten belastet. Allerdings führen die Sozialversicherungsbeiträge im Vergleich zu Deutschland, wo sie rund $40 \%$ des Bruttolohns betragen, zu einer wesentlich stärkeren Abgabenbelastung des Faktors Arbeit. So belaufen sich in Frankreich die Abgaben für die Sozialversicherungen von Arbeitnehmern und Arbeitgebern im Jahr 2008 insgesamt auf über $60 \%$ des Bruttolohns, wobei davon rund ein Drittel auf arbeitnehmer- und zwei Drittel auf arbeitgeberseitige Abgaben zu den Sozialversicherungen entfallen. ${ }^{(\infty}$ Während man diesem Fakt in Deutschland im Bereich der Geringverdiener durch die Einführung von Mini- und Midijobs begegnen will, subventioniert Frankreich zum einen die arbeitgeberseitigen Sozialversicherungsbeiträge im unteren Einkommensbereich zu einem gewissen Teil aus Steuermitteln. Bei einem Einkommen in Höhe des französischen Mindestlohns, d.h. beispielsweise im Falle einer Vollzeittätigkeit von 1.820 Stunden bei einem jährlichen Einkommen von $15.670 €($ OECD 2009, S. 234), ist die arbeitgeberseitige Lohnsubvention am größten, sodass die von Arbeitgebern und Arbeitnehmern getragenen Abgaben zu den Sozialversicherungen lediglich bei knapp $38 \%$ liegen. Danach wird die arbeitergeberseitige Subvention der Sozialversicherungsbeiträge mit zunehmendem Lohneinkommen linear mit einer Entzugsrate von $43 \%$ bis zu einem jährlichen Einkommen in Höhe des 1,6-Fachen des gesetzlichen Mindestlohns abgebaut. Infolgedessen steigen die von Arbeitgebern und Arbeitnehmern getragenen Abgaben zu den Sozialversicherungen auf rund $64 \%$ im gleichen Intervall an. Ohne diese Subventionierung würden auch Geringverdiener mit einer Belastung von über $60 \%$ des Mindestlohns einsteigen. Laut Sterdyniak (2007, S. 18) kostete die Subventionierung der Sozialversicherungsbeiträge im Jahr 2006 knapp 18 Mrd. € oder $1 \%$ des Bruttoinlandsprodukts (BIP).

Zum anderen wird diese arbeitgeberseitige Subvention durch eine Beschäftigungsprämie für Bezieher niedriger
Einkommen, den Prime pour l'emploi (PPE) ergänzt. Diese Beschäftigungsprämie ist ein arbeitnehmerseitiger Lohnzuschuss, der aus individueller Sicht aber einen eher geringen finanziellen Umfang hat. Zwar wurde die maximale PPE-Höhe seit 2005 fast verdoppelt und lag 2008 bei $961 €$ im Jahr (Duval 2009, S. 2), sodass sie im unteren Einkommensbereich den Charakter eines vollständigen 13. Monatsgehalts erhalten hat, sie ist aber in Bezug auf ihre finanzielle Ausgestaltung viel niedriger als die Steuergutschrift für Erwerbstätige im Vereinigten Königreich. Die seit 2001 bestehende PPE richtet sich an Personen im Niedrigeinkommenssegment, die wieder eine Arbeit aufnehmen bzw. schon beschäftigt sind, und zwar unabhängig davon, ob sie einer Vollzeit- oder Teilzeitbeschäftigung nachgehen; sie richtet sich aber auch an Selbstständige. Ihre Höhe wird abhängig vom Einkommen festgelegt. Sie wird von der Einkommensteuer abgezogen oder bei einer Nichtveranlagung wegen zu geringen Einkommens - so der Regelfall - direkt monatlich ausgezahlt. Die Berechnung der PPE ist komplex und erfolgt in mehreren Schritten. Zunächst wird für jede anspruchsberechtigte erwerbstätige Person eines Haushalts eine Beschäftigungsprämie ermittelt, diese werden dann addiert und unter bestimmten Umständen (beispielsweise bei Existenz eines unterhaltspflichtigen bzw. finanziell noch abhängigen Kindes oder bei einer alleinerziehenden Person) noch um gewisse Festbeträge erhöht. Diese Summe wird von der Steuerschuld des Haushalts abgezogen, sofern das zu versteuernde Haushaltseinkommen gewisse Höchstbeträge nicht übersteigt. Ähnlich wie im Vereinigten Königreich mit der Mindestanzahl an zu leistenden Arbeitsstunden gibt es auch in Frankreich eine Mindestförderschwelle, die hier jedoch mittels eines Mindestarbeitseinkommens definiert wird. Anspruch auf die PPE haben demnach Erwerbstätige mit einem jährlichen Arbeitseinkommen, das mindestens dem 0,3-Fachen eines fiktiven Vollzeiteinkommens mit einem Stundenlohn in Höhe des französischen Mindestlohns SMIC entspricht. Ab dieser Schwelle bis zu einem Jahreseinkommen in Höhe des SMIC haben Berechtigte dann einen Anspruch in Höhe von 7,7\% des individuellen Arbeitseinkommens, anschließend wird die Beschäftigungsprämie gleitend bis auf null zurückgeführt (Abbildung 2). Teilzeitbeschäftigte werden seit 2003 besonders gefördert. So erhielt 2008 eine Person, die ein Arbeitsvolumen von $50 \%$ einer Vollzeitstelle hatte und entsprechend dem Mindestlohn bezahlt wurde, 92,5\% der Beschäftigungsprämie, die der Vollzeitstelle entsprochen hätte (Duval 2009, S.2). 2008 gab es insgesamt 8,9 Mio. Haushalte oder $25 \%$ aller Steuerhaushalte, die die Beschäftigungsprämie bezogen. Die Gesamtkosten betrugen insgesamt etwa 4,5 Mrd. € (Duval 2009, Tabelle 2).

๑ Im Jahr 2011 liegt der SMIC bei $9 €$.

(1) Für Details siehe Sterdyniak 2007, Tabelle 5 oder OECD 2009, S. $227 \mathrm{ff}$. 
ABB. 2

\section{Beschäftigungsprämie in Frankreich (prime pour l'emploi, PPE) 2008}

- Einzelperson Einverdienerhaushalt

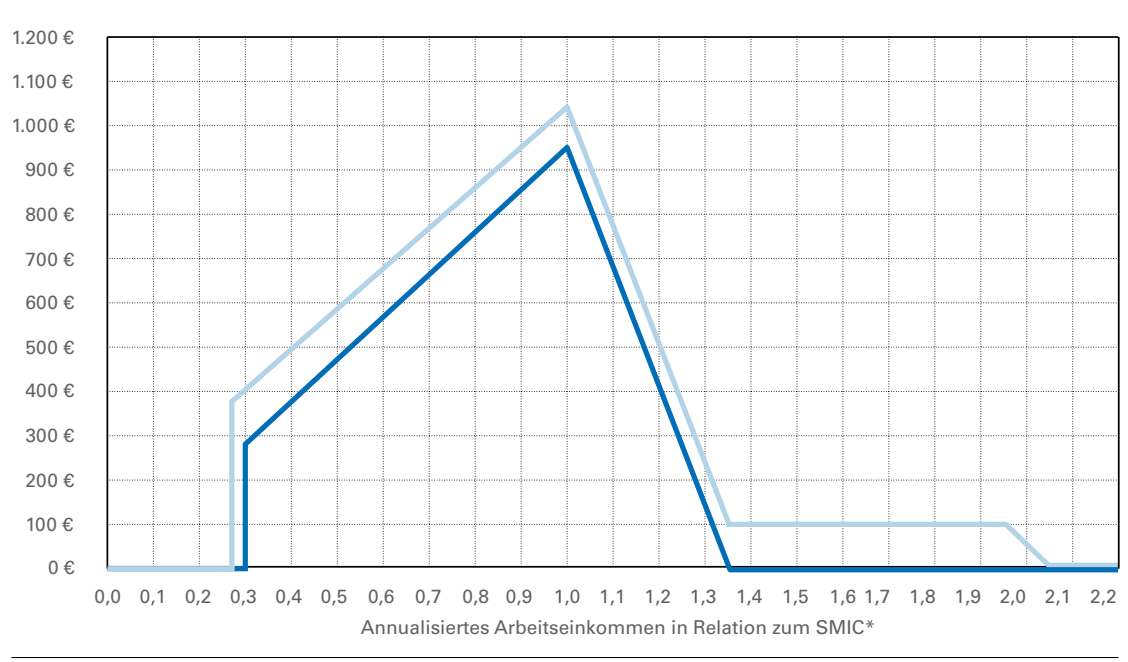

*Salaire minimum interprofessionnel de croissance (gesetzlicher Mindestlohn).

In Bezug auf die gesamte Abgabenbelastung im unteren Einkommensbereich ist natürlich auch die Ausgestaltung der Einkommensteuer relevant. Laut den von Dingeldey (2002) identifizierten Typen der Familienbesteuerung hat Frankreich, ebenso wie Deutschland, ein Steuersystem mit gemeinsamer Ehegattenbesteuerung. Allerdings gibt es in Frankreich anstelle des Ehegattensplittings ein Familiensplitting. „Hierbei wird die Zahl aller Familienmitglieder einbezogen, indem das Familieneinkommen durch die Zahl der Haushaltsangehörigen geteilt, die entsprechende Steuerrate angewendet und dann wieder mit der Zahl der Haushaltsangehörigen multipliziert wird“" (Dingeldey 2002, S. 155). Der Steuerfreibetrag lag 2008 bei $5.687 €$ pro erwerbstätige Person. Darüber greift zunächst der Eingangssteuersatz von 5,5\%. Mit zunehmendem Einkommen steigt der Grenzsteuersatz progressiv bis auf $40 \%$ für Jahreseinkommen von mehr als $67.564 €$ (OECD 2009, S. 227ff.).

Als Zwischenfazit kann festgehalten werden, dass insbesondere die Doppelstrategie aus arbeitgeber- und arbeitnehmerseitigen Lohnsubventionen am französischen Steuer- und Transfersystem im unteren Einkommensbereich und die Ausrichtung der einzelnen Instrumente am allgemeinen gesetzlichen Mindestlohn ins Auge stechen. Der finanzielle Schwerpunkt liegt auf den Lohnsubventionen für Arbeitgeber, während die Beschäftigungsprämie, der Lohnzuschuss für Arbeitnehmerinnen und Arbeitnehmer mit niedrigem Einkommen, eher einen geringen finanziellen Umfang aufweist. Hervorzuheben ist zudem, dass - wie im Vereinigten Königreich - Beschäftigungsverhältnisse erst explizit gefördert werden, wenn sie oberhalb einer Mindestförderschwelle liegen.

\section{Beschäftigungs- und Einkommens- effekte im Vereinigten Königreich und in Frankreich}

Welche Steuerungs- und Anreizwirkungen haben die skizzierten Regulierungen in den beiden betrachteten Ländern? Neben der Einkommensentwicklung stellt sich insbesondere die Frage nach der Beschäftigungsentwicklung in den zu betrachtenden Segmenten.

\subsection{Beschäftigungseffekte}

Für das Vereinigte Königreich haben sich mehrere Untersuchungen mit der Frage nach den Beschäftigungseffekten der Steuergutschriften, wie sie zwischen 1999 und 2003 existierten, beschäftigt. Brewer/Browne (2006) präsentieren einen detaillierten Literaturüberlick. Danach stimmen die verschiedenen Studien darin überein, dass sich die Zahl der geleisteten Arbeitsstunden von Alleinerziehenden im Durchschnitt erhöht hat. Je nach Studie gibt es aber erhebliche Unterschiede im Hinblick auf das geschätzte Ausmaß des Beschäftigungseffekts. Darüber hinaus gibt es Hinweise, dass bei Ehepaaren mit Kindern sowohl der Anteil der Zweiverdienerhaushalte als auch der der erwerbslosen Haushalte zurückgegangen ist. Laut Brewer/Browne (ebd.) ist es aber wahrscheinlich, dass sich die Beschäftigungsquote insgesamt erhöht hat, da der erste Effekt kleiner ausfiel als der letzte.

Im Hinblick auf die direkten Beschäftigungseffekte des WTC gibt es unseres Wissens nach keine Untersuchungen. Blundell et al. (2004) untersuchen die Effekte aller Veränderungen des Steuer- und Transfersystems im Zeitraum der Jahre 2000 bis 2003 mit Hilfe eines mikroökonometrischen Simulationsmodells. Sie sagen eine Zunahme der Partizipationsquote von alleinstehenden Müttern um 3,38\% sowie einen Rückgang der Partizipationsquote von verheirateten Müttern um 0,35\% und bei verheirateten Vätern eine Zunahme um 0,92\% voraus. Zudem wird ein Rückgang der Arbeitsstunden bei berufstätigen Eltern vorhergesagt.

$\mathrm{Zu}$ Frankreich befassten sich zahlreiche Untersuchungen mit den Beschäftigungseffekten der reduzierten Arbeitgeberbeiträge zu den Sozialversicherungen. Zwar schwanken die ermittelten Beschäftigungseffekte durchaus erheblich, aber insgesamt scheint ein Konsens darüber zu bestehen, dass die Subventionen die Beschäftigung von Niedrigqualifizierten erhöht haben (Lindner 2010). So vergleicht Sterdyniak (2007) insgesamt 15 Studien zu den Beschäftigungseffekten der reduzierten Arbeitgeberbeiträge, wonach sich der geschätzte Effekt auf ungefähr 300.000 Arbeitsplätze infolge einer Beitragsreduktion im Umfang von (ex ante) 10 Mrd. $€$ beläuft. Auf Basis von geschätzten Gesamtausgaben von $18 \mathrm{Mrd}$. $\mathrm{im}$ Jahr 2007 würde demnach ein auf diesem Weg geschaffener zusätzlicher Arbeitsplatz ex post $17.600 €$ kosten (Sterdyniak 2007, S. 18). 
Der Beschäftigungsprämie PPE werden nur kleine positive Beschäftigungseffekte etwa in Höhe von 0,5 Prozentpunkten zugerechnet (vgl. Sterdyniak 2007; Immervoll/ Pearson 2009). Die Ursachen hierfür werden in dem relativ geringen individuellen Subventionsumfang, der allgemein schwierigen Arbeitsmarktsituation, den komplexen Regeln und den langen Fristen zwischen der Anspruchsberechtigung und der Auszahlung gesehen (Immervoll/Pearson 2009, S. 29).

Wird die Beschäftigungsstruktur in den drei Ländern miteinander verglichen, so zeigen sich deutliche Unterschiede. Im Vereinigten Königreich - wie in Deutschland arbeitet ein erheblicher Teil der erwerbsfähigen Bevölkerung in marginaler Teilzeit. Nach Eichhorst et al. (2010, Abbildung 3) lag der Anteil der marginal Teilzeitbeschäftigten 2006 im Vereinigten Königreich bei 3,6\%, während er in Deutschland bei 4,2\% lag. In Frankreich spielt diese Beschäftigungsform dagegen mit einem Anteil an der erwerbsfähigen Bevölkerung von 1,0\% nur eine geringe Rolle. Es scheint somit nicht zu den in Deutschland beobachtbaren Lock-in-Effekten gekommen zu sein. Die arbeitgeber- wie arbeitnehmerseitigen Lohnsubventionen haben nicht zu Anreizen in die kurze Teilzeit geführt, wie im Falle von Minijobs. In Bezug auf das Vereinigte Königreich ist das Ergebnis zunächst überraschend, da die britischen Steuergutschriften aufgrund der Mindestförderschwelle gerade keine Beschäftigung im Bereich der marginalen Teilzeit fördern. Eine mögliche Ursache könnten aber die relativ großzügigen Freibeträge bei den Sozialversicherungsbeiträgen sein, die dazu führen könnten, dass „sozialversicherungsfreie“ Teilzeitjobs für Beschäftigte und Arbeitgeber attraktiv sind.

\subsection{Einkommenseffekte}

In den drei Ländern zeigen sich interessante Unterschiede in der Einkommensspreizung. So erhöhte sich die Einkommensspreizung ${ }^{\oplus}$ in Deutschland zwischen den mittleren und unteren Einkommen ab Mitte der 1990er Jahre bis 2005, während in Frankreich genau die gegenteilige Entwicklung festzustellen ist (Immervoll/Pearson 2009, S. 12; Eichhorst et al. 2010, S. 32). Erstaunlicherweise zeigt sich für das Vereinigte Königreich nahezu keine Veränderung, denn das Verhältnis der Einkommen im mittleren Einkommensdezil zu den Einkommen im unteren Dezil blieb über die Jahre hinweg auf etwa dem gleichen Niveau. Demgegenüber stieg die Differenz zwischen den mittleren Einkommen in Deutschland und den unteren im Betrachtungszeitraum deutlich an, und inzwischen hat Deutschland eine größere Einkommensspreizung in der unteren Hälfte der Einkommensverteilung nicht nur als Frankreich, sondern auch als das Vereinigte Königreich (Abbildung 3).

Insbesondere $\mathrm{ab}$ der Jahrtausendwende lässt sich hier eine stark divergierende Entwicklung vor allem zwischen Deutschland und Frankreich feststellen, was umso erstaunlicher ist, da beide Länder üblicherweise den gleichen Wohl-
ABB. 3

\section{Einkommensverteilung im unteren Bereich in Deutschland, dem Vereinigten Königreich und Frankreich 1994 - 2008}

Entwicklung des Verhältnisses der Perzentile 50 und 10 bei den Bruttoeinkommen der abhängig Beschäftigten in Vollzeit - Vereinigtes Königreich Deutschland Frankreich

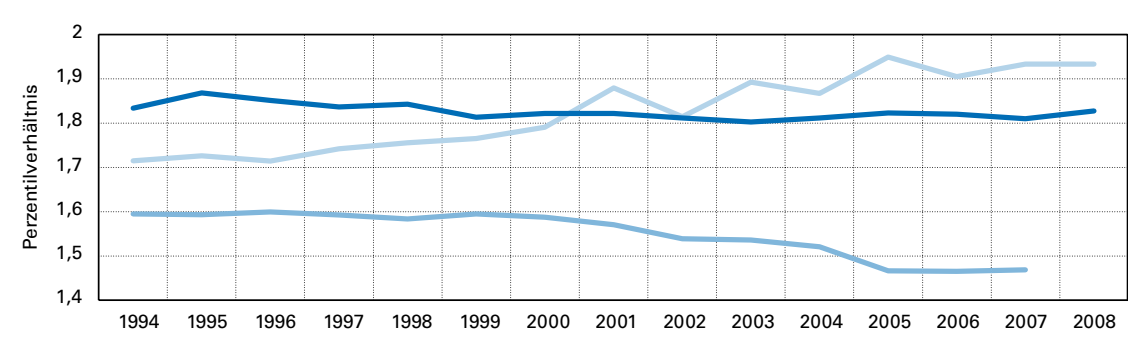

Quelle: OECD; Berechnung und Darstellung der Autoren.

fahrtsstaatstypen mit analogen Problemen zugeordnet werden. So stieg das 50/10-Verhältnis in Deutschland um mehr als $12 \%$. In Frankreich war dagegen ein Rückgang um knapp $8 \%$ zu beobachten. Eine erhebliche Rolle dürfte dabei die Tatsache gespielt haben, dass sowohl in Frankreich als auch im Vereinigten Königreich die Lohnzuschüsse im unteren Einkommensbereich durch einen allgemeinen gesetzlichen Mindestlohn ergänzt werden, während dies in Deutschland nicht der Fall ist.

\section{Schlussfolgerungen}

Wie Deutschland so haben auch das Vereinigte Königreich und Frankreich das Steuer- und Abgabensystem im unteren Einkommensbereich mit der Absicht umgestaltet, die Abgabenbelastung niedriger Einkommen zu reduzieren und die damit verbundenen Anreize zur Arbeitsaufnahme zu erhöhen sowie die Einkommenssituation dieser Haushalte zu verbessern. Obwohl alle drei Länder hierfür Lohnzuschüsse nutzen, gibt es erhebliche Unterschiede zwischen den gewählten Strategien.

In Bezug auf die arbeitnehmerseitigen Lohnzuschüsse gibt es sowohl im Vereinigten Königreich als auch in Frankreich eine Mindestförderschwelle in Form einer Min-

(1) In der OECD-Datenbank stehen nur Informationen auf Grundlage der Bruttoverdienste von abhängig Beschäftigten in Vollzeit zur Verfügung. Es ist aber davon auszugehen, dass bei Berücksichtigung derTeilzeitbeschäftigten die Ergebnisse qualitativ fortbestehen. 
destzahl an Arbeitsstunden bzw. eines Mindesteinkommens. Dadurch wird sichergestellt, dass keine marginalen Beschäftigungsverhältnisse, sondern nur Arbeitsplätze mit einem gewissen Arbeitsumfang gefördert werden. Dies ist das genaue Gegenteil der deutschen Mini- und Midijobregelungen, die nur für Beschäftigungsverhältnisse unterhalb definierter Einkommensgrenzen Anreize setzen. Schließlich erfolgt in beiden Ländern die direkte Förderung niedriger Arbeitseinkommen mit den Lohnzuschüssen WTC und PPE über das Steuersystem.

Weniger konsequent sind das Vereinigte Königreich und Frankreich hinsichtlich der Zielwirkung der Entlastung von Sozialversicherungsbeiträgen im unteren Einkommensbereich. Im Vereinigten Königreich sind wöchentliche Einkommen von weniger als $105 £(132 €)$ für Arbeitnehmer und Arbeitgeber von Zahlungen an die Sozialversicherungen befreit. Dies könnte eine mögliche Ursache dafür sein, dass es im Vereinigten Königreich, wie in Deutschland, einen beträchtlichen Anteil an marginal Beschäftigten gibt. In Frankreich dagegen existieren weder Freibetrag noch -grenze in den Sozialversicherungen. Stattdessen werden die arbeitgeberseitigen Sozialversicherungsbeiträge subventioniert. Zwar gibt es auch hier eine Mindestfördergrenze durch den französischen Mindestlohn, allerdings werden auch Teilzeitarbeitsplätze subventioniert - wenn auch nur anteilig entsprechend dem gearbeiteten Arbeitsvolumen. Insgesamt scheint das französische System im unteren Einkommensbereich aber weniger Anreize zur marginalen Beschäftigung zu bieten als die Abgabensysteme in Deutschland und dem Vereinigten Königreich.

Sowohl in Frankreich als auch dem Vereinigten Königreich - nicht aber in Deutschland - existiert ein allgemeiner gesetzlicher Mindestlohn und setzt damit klare Untergrenzen für Stundenlöhne. Die Existenz eines allgemeinen Mindestlohns reduziert die Traglastproblematik der Sozialversicherungsbeiträge, wie sie in Deutschland zu beobachten ist, erheblich. Darüber hinaus liefert die Entwicklung der Einkommensungleichheit in den drei Ländern Anhaltspunkte dafür, wie wichtig ein Mindestlohn für die Stabilisierung der Einkommensverteilung ist.

Die Analyse der Lohnzuschussmodelle Frankreichs und des Vereinigten Königreichs geben Hinweise darauf, dass es durchaus effektivere Maßnahmen als die deutschen Miniund Midijobregelungen gibt, um die Beschäftigungs- und Einkommenssituation der Haushalte im Bereich niedriger Einkommen zu verbessern. So könnten durch die Einführung einer direkten Förderung niedriger Erwerbseinkommen in Form von Lohnzuschüssen über das Steuersystem und einer Mindestförderschwelle in Verbindung mit einem allgemeinen Mindestlohn die für Deutschland festzustellenden Unterschiede der Bruttolöhne von Minijobbern und sozialversicherungspflichtigen Erwerbstätigen minimiert werden. Zudem würde die Einführung einer Mindestförderschwelle beispielsweise in Höhe von 15 Erwerbsstunden pro Woche die lock-in-Effekte in sehr kleinen Beschäftigungsverhältnissen vermeiden und dazu führen, dass die Trans- ferbezieher nicht mehr als Arbeitslose geführt werden. Darüber hinaus hätte all dies zwei weitere entscheidende Vorteile: Zum einen könnten einheitliche Sozialversicherungsbeiträge ab dem ersten Euro erhoben werden, womit der Sonderstatus von Minijobs abgeschafft würde. Und zum anderen würde die Förderung von Erwerbstätigen nicht mehr mit der Alimentierung von Arbeitsuchenden verquickt. Gerade Letzteres sollte die individuelle und kollektive Wertschätzung von Lohnzuschussbeziehern im Vergleich zur jetzigen Situation erhöhen. 


\section{LITERATUR}

Apel, H./Burda, M. C./Buscher, T./Claus, T./Fertig, M./Friedrich, W./Haegele, H./Jacobi, L./Kluve, J./Kvasnicka, M./Schaffner, S./Schmidt, C. M./Schumacher, P./Tamm, M. (2006): Evaluation der Maßnahmen zur Umsetzung der Vorschläge der Hartz-Kommission - Modul 1f: Verbesserung der beschäftigungspolitischen Rahmenbedingungen und Makrowirkungen der aktiven Arbeitsmarktpolitik, Forschungsbericht des Bundesministeriums für Arbeit und Soziales (BMAS), Berlin

Bargain, O./Orsini, K. (2006): In-work policies in Europe: Killing two birds with one stone?, in: Labour Economics 13 (6), S. 667-697

Blundell, R./Brewer, M./Shepard, A. (2004): The impact of tax and benefit changes between April 2000 and April 2003 on parents' labour supply, Institute for Fiscal Studies (IFS), IFS briefing note (52)

Brewer, M./Browne, J. (2006): The Effect of the Working Families' Tax Credit on Labour Market Participation, Institute for Fiscal Studies (IFS), IFS briefing note (69)

Bundesministerium für Wirtschaft und Arbeit (BMWA) (2003): Brücken in den Arbeitsmarkt - Wirtschaftsbericht 2003, Berlin, http://www.bmwi.de/Redaktion/Inhalte/Downloads/wirtschaftsbericht-03,property=pdf.

Bundesregierung (2003): Bericht der Bundesregierung zu den Auswirkungen des Gesetzes zur Neuregelung der geringfügigen Beschäftigungsverhältnisse auf den Arbeitsmarkt, die Sozialversicherung und die öffentlichen Finanzen,

Deutscher Bundestag, Drucksache 15/758

Dingeldey, I. (2002): Das deutsche System der Ehegattenbesteuerung im europäischen Vergleich, in: WSI-Mitteilungen 55 (3), S. 154-160

Duval, J. (2009): The French employment premium and its beneficiaries, 20012008, Ministère de l'Économie, de l'Industrie et de l'Emploi, Direction Générale du Trésor et de la Politique économique, Trésor Economics (63), July, Paris

Eichhorst, W./Marx, P./Thode, E. (2010): Atypische Beschäftigung und Niedriglohnarbeit, IZA Research Report (25), Gütersloh

Eichhorst, W./Thode, E. (2010): Vereinbarkeit von Familie und Beruf 2010 Benchmarking Deutschland: Steigende Erwerbsbeteiligung, aber schwierige Übergänge, IZA Research Report (30), Bonn

HM Revenue and Customs (2009): HM Revenue \& Customs 2008-09 Accounts, London, http://www.hmrc.gov.uk/about/hmrc-accs-0809.pdf Immervoll, H./Pearson, M. (2009): A GoodTime for Making Work Pay? Taking Stock of In-Work Benefits and Related Measures across the OECD, IZA Policy Paper (3), Bonn

Kaltenborn, B./Knerr, P. (2005): Arbeitsanreize im Niedriglohnbereich - ein ausgewählter internationaler Vergleich, Gutachten im Auftrag des Bundesministeriums für Arbeit und Soziales, Berlin
Lindner, F. (2010): Die Beschäftigungseffekte der französischen Arbeitszeitverkürzung und der Lohnsubventionen - eine Literaturübersicht, Düsseldorf Nicholson, W. (1998): Microeconomic Theory - Basic Principles and Extensions, Fort Worth

Organisation for Economic Co-operation and Development (OECD) (2009): Taxing Wages 2007-2008, Paris

Sachverständigenrat zur Begutachtung der gesamtwirtschaftlichen Entwicklung (SVR) (2006): Arbeitslosengeld II reformieren: Ein zielgerichtetes Kombilohnmodell, Expertise im Auftrag des Bundesministers für Wirtschaft und Technologie, August, Wiesbaden

Sterdyniak, H. (2007): Low-skilled Jobs: The French Strategy, Obsertoire Francais des Conjonctures Économiques (OFCE), Document de travail 2007-15, Juli, Paris

Strickland, P. (1998): Working Families Tax Credit and Family Credit, House of Commons Research Paper 98/46, 9. April, London

Wingerter, C. (2009): Der Wandel der Erwerbsformen und seine Bedeutung für die Einkommenssituation Erwerbstätiger, in: Wirtschaft und Statistik (11), S. 1080-1098

Weinkopf, C. (2011): Minijobs - politisch-strategische Handlungsoptionen, Expertise, Universität Duisburg-Essen

\section{AUTOREN}

ALEXANDER HERZOG-STEIN, Dr., Wissenschaftler im Wirtschafts- und Sozialwissenschaftlichen Institut (WSI) in der Hans-Böckler-Stiftung. Arbeitsschwerpunkte: Arbeitsmarkt- und Arbeitszeitforschung.

alexander-herzog-stein @ boeckler.de

WERNER SESSELMEIER, Prof. Dr., Leiter der Abteilung Wirtschaftswissenschaft an der Universität Koblenz-Landau, Campus Landau und federführender Editor der Zeitschrift Sozialer Fortschritt. Arbeitsschwerpunkte: Arbeitsmarktund Sozialpolitik.

sesselmeier@uni-landau.de 